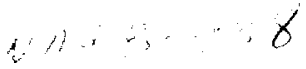

$$
\begin{aligned}
& \text { iN-3\%-CH } \\
& \because 20 \%
\end{aligned}
$$
\title{
Effect of Baffle on Gravity-Gradient-Excited Slosh Waves
and Spacecraft Moment and Angular-Momentum Fluctuations in Microgravity.
}

\author{
R. J. HUNG and C. C. LEE \\ The University of Alabama in Huntsville - Huntsville, AL 35899, USA
}

(ricevuto il 31 Gennaio 1994; revisionato il 9 Marzo 1995; approvato il 31 Marzo 1995)

Summary. - The dynamical behavior of fluids affected by the asymmetric gravity gradient acceleration has been investigated. In particular, the effects of surface tension on partially filled rotating fluids applicable to a full-scale Gravity Probe-B Spacecraft dewar tank with and without baffles are studied. Results of slosh wave excitation along the liquid-vapor interface induced by gravity gradient acceleration indicate that the gravity gradient acceleration is equivalent to the cort. The results are a twisting force and a torsional moment acting on the-up oscillations in the crossclearly seen from one-up one-down and one-down on section profiles of two bubbles in the vertical $(r, z)$-plane the axis of the dewar in a from the eccentric contour of the bubble rotating between liquid and solid interface, horizontal $(r, \theta)$-plane. As the viscous slosh wave excitation, a rotating dewar with greatly contributes to the damping of slosh wavece than that of a rotating dewar baffles provides more areas of liquid-solid inteffect provided by the baffles reduces without baffles. Results show that the damp the amplitude of slosh wave excitation and lower thomentum and fluid moment liquid-vapor distribution. Fluctuations of angularent acceleration with and without caused by the slosh wave excited by gravity gradient at the damping effect provided baffle boards are also investigated. It is also shown that the dantum and fluid moment by the baffles

of relativity and observations of gravita-

PACS 04.80 - Experimental tests of general
tional radiation.

PACS 47.20.Dr - Surface-tension-driven instability.

\section{1. - Introduction.}

Some experimental spacecrafts use superconducting sensors for gyro read-out and very low temperature for mechanical stability. The approaches to both cooling and control involve the use of superfluid liquid helium. For example, the Gravity Probe-B (GP-B) Spacecraft adopts the boil-off from the cryogenic-liquid- 


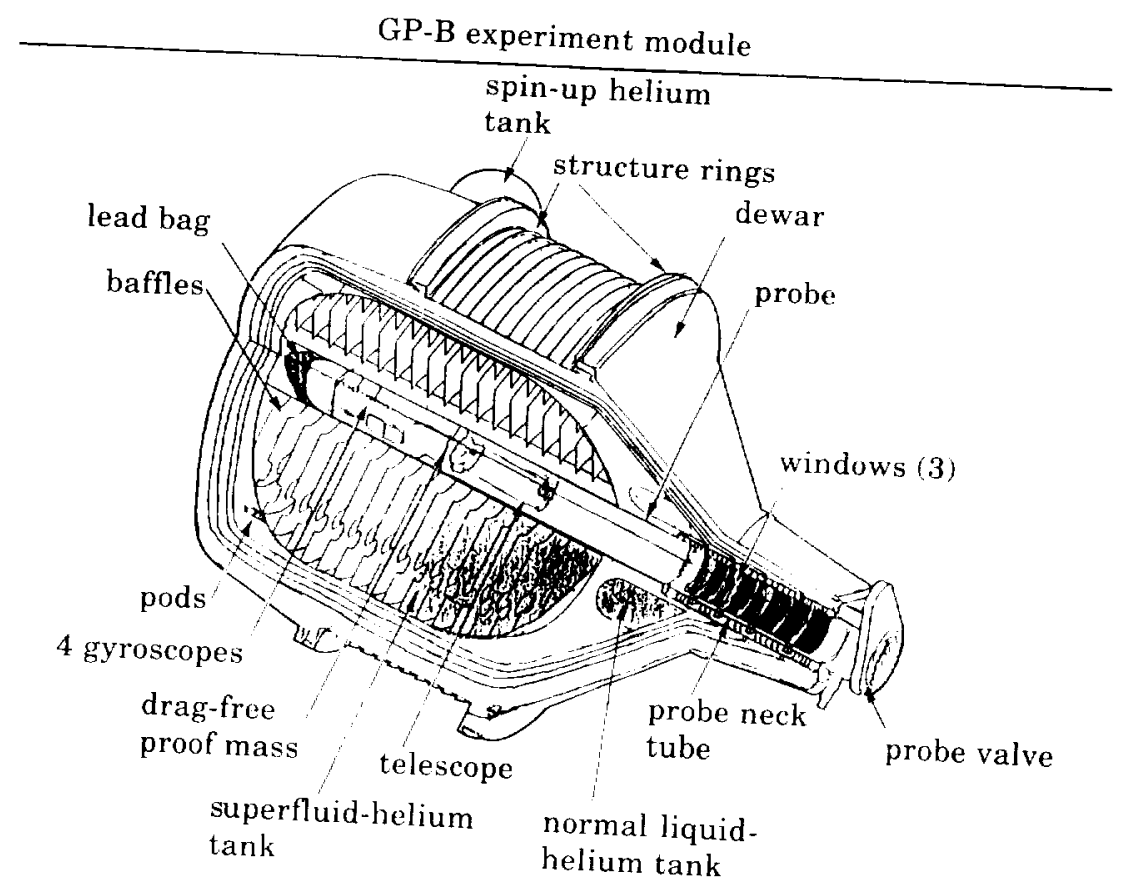
Fig. 1. - The experiment Gravity Probe-B module showing the main elements of liquid-helium
dewar, probe and baffle boards.

helium dewar as a coolant and a propellant to maintain the attitude control and in the dewar container (fig. 1) could be. The potential problems for cryogenic liquid distribution or to perturbations in be due to asymmetry in the static liquid-helium baffle-board inside the dewar container (as shown. It may be necessary to install a asymmetry in the liquid-vapor distribution.

For the case of the

of $1.8 \mathrm{~K}$ is used as the propellant. Withogenic liquid helium II at a temperature temperature gradients in the liquid helium its superfluid behavior, there are no along the surfaces which drives Marangoni. In the absence of temperature gradient free surface is governed by a balance of cani convection, the equilibrium shape of the Determination of vapor profiles based capillary, centrifugal and gravitational forces. details of the flow which can not be easily computational experiments can uncover microgravity environment.

The instability of the

and lateral accelerations, vehich lice can be induced by the presence of longitudinal Slosh waves are, thus, excited and protion, and rotational fields of the spacecraft. the liquid systems. The sources of the residuagh- and low-frequency oscillations in the Earth's gravity gradient, atmospherial accelerations range from the effects of attitude motions arising from machinery drag on the spacecraft, and spacecraft motion. A recent study [2] suggests that the vibrations, thruster firings, and crew unimportant in comparison to the residua high-frequency accelerations may be motions caused by low-frequency 
Time-dependent dynamical behavior of rotating fluids in reduced-gravity environments was simulated by numerically solving the Navier Stokes equations subject to initial and boundary conditions [3-5]. At the interface between the liquid and the gaseous fluids, both the kinematic surface boundary condition, and the interface stress conditions for components tangential and normal to the interface, were applied [3-5]. The initial conditions were adopted from the steady-state formulations developed by Hung et al. [3] in the geometry of the GP-B Spacecraft [1]. Some of the steady-state formulations of interface shapes were compared with the available experiments carried out by Leslie [6] in a free-falling aircraft ( $\mathrm{KC}-135)$. The experiments carried out by Mason et al. [7] showed that the classical fluid mechanics theory is applicable to cryogenic liquid helium in velocities greater than critical velocity and in the large containers [8].

As to the effect of centrifugal force on the liquid helium and helium vapor, the dewar tank will not be spinning when the GP-B Spacecraft is deployed. In the early stages of the experiment, a spin rate of up to about 1 r.p.m. will be imposed for instrument calibration. After calibration, the rotation rate will be reduced to its operational value of approximately 0.1 r.p.m.

In the spacecraft orbit around the Earth, the direction of the azimuth angle from Earth toward the location of the spacecraft mass center varies from $0^{\circ}$ along the axis of rotation to $360^{\circ}$ directions in which axial symmetry is no more applicable and a three-dimensional calculation shall be adopted.

As the spacecraft moves along the orbit, any fluid capable of motion relative to the spacecraft is subject to the acceleration that arises from the gravity gradients of the Earth [9-11]. The interaction between the mass of the fluid particles and the spacecraft mass due to gravity gradient acceleration [10] can excite slosh waves. In this paper, studies are made for the dynamical evolution of slosh wave excited by the driving forces due to gravity gradient acceleration.

In order to reduce the degree of asymmetry in the liquid-vapor distributions which is induced by asymmetric gravity-gradient-acceleration-excited slosh waves, a number of baffle boards are installed inside the dewar (as shown in fig. 1). Comparison will be made between the degree of asymmetry in liquid-vapor distribution for a dewar with and without baffles during the time period of slosh wave excitation induced by asymmetric gravity gradient acceleration acting on the spacecraft. The time evolution of the profiles of liquid-vapor interface which disturb moment and angular momentum of fluid systems for a rotating dewar container with and without baffle boards is investigated.

\section{2. - Mathematical model of slosh wave excitation along the liquid-vapor interface.}

Consider a closed circular dewar of inner radius $a_{i}$, outer radius $a_{0}$, top and bottom dome of radius $b$, with height $L$, which is partially filled with cryogenic liquid helium, and with the rest of the ullage filled with a helium vapor. The density and viscosity of liquid helium and helium vapor are $\varrho_{\mathrm{L}}, \mu_{\mathrm{L}}, \varrho_{\mathrm{v}}$ and $\mu_{\mathrm{v}}$, respectively. Let us use cylindrical coordinates $(r, \theta, z)$, with corresponding velocity components $(u, v, w)$, and corresponding Coriolis force $(2 \varrho \omega v,-2 \varrho \omega u, 0)$, centrifugal force $\left(\varrho r \omega^{2}, 0,0\right)$, angular acceleration $(0$, or $\dot{\omega}, 0)$ and gravity gradient acceleration $\left(a_{\mathrm{gg}, r}, a_{\mathrm{gg}, \theta}, a_{\mathrm{gg} . z}\right)$ for a dewar rotating with angular velocity $(0,0, \omega)$. The governing equations for non-inertial frame of spacecraft-bound coordinates can be shown as follows: 
A) continuity equation

$$
\frac{1}{r} \frac{\partial}{\partial r}(r u)+\frac{1}{r} \frac{\partial v}{\partial \theta}+\frac{\partial w}{\partial z}=0,
$$

B) momentum equations

$$
\begin{aligned}
& \varrho\left(\frac{\partial u}{\partial t}+u \frac{\partial u}{\partial r}+\frac{v}{r} \frac{\partial u}{\partial \theta}-\frac{v^{2}}{r}+w \frac{\partial u}{\partial z}\right)=-\frac{\partial p}{\partial r}+2 \omega u v+\varrho\left(a_{\mathrm{gg}, r}+r \omega^{2}\right)+ \\
& +\mu\left(\nabla^{2} u-\frac{u}{r^{2}}-\frac{2}{r^{2}} \frac{\partial v}{\partial \theta}\right)
\end{aligned}
$$

$$
\begin{gathered}
\varrho\left(\frac{\partial v}{\partial t}+u \frac{\partial v}{\partial r}+\frac{v}{r} \frac{\partial v}{\partial \theta}+\frac{u v}{r}+w \frac{\partial v}{\partial z}\right)=-\frac{1}{r} \frac{\partial p}{\partial \theta}-2 \varrho\left(v u+\varrho\left(a_{g \mathrm{~g}, \theta}+r \dot{\omega}\right)+\right. \\
+\mu\left(\nabla^{2} v-\frac{v}{r^{2}}+\frac{2}{r^{2}} \frac{\partial u}{\partial \theta}\right) \\
\varrho\left(\frac{\partial w}{\partial t}+u \frac{\partial w}{\partial r}+\frac{v}{r} \frac{\partial w}{\partial \theta}+w \frac{\partial w}{\partial z}\right)=-\frac{\partial p}{\partial z}+\varrho\left(a_{\mathrm{kg} . z}\right)+\mu \nabla^{2} w
\end{gathered}
$$

where

$$
\nabla^{2}=\frac{1}{r} \frac{\partial}{\partial r}\left(r \frac{\partial}{\partial r}\right)+\frac{1}{r^{2}} \frac{\partial^{2}}{\partial \theta^{2}}+\frac{\partial^{2}}{\partial z^{2}} .
$$

In the computation of fluid forces, moment, viscous stress and angular momentum acting on the container wall of the spacecraft, one has to consider the velocity in the inertial frame rather than in the non-inertial frame. In this particular case, one has to transform these vectors from the non-inertial frame to the inertial frame of the spinning motion in the $z$-axis.

$$
\left(\begin{array}{l}
F_{y}^{\prime} \\
F_{y}^{\prime} \\
F_{z}^{\prime}
\end{array}\right)=\left(\begin{array}{ccc}
\cos \omega t & -\sin \omega t & 0 \\
\sin \omega t & \cos \omega t & 0 \\
0 & 0 & 1
\end{array}\right)\left(\begin{array}{l}
F_{y} \\
F_{y y} \\
F_{z}
\end{array}\right),
$$

where the prime symbol denotes vectors in the inertial frame while those parameters without the prime symbols indicate vectors in the non-inertial frame.

For the purpose of solving sloshing-dynamic problems of liquid systems in orbital spacecraft under a microgravity environment, one has to solve the governing equations (1) to (5) accompanied by a set of initial and boundary conditions. A detailed illustration of these initial and boundary conditions concerning sloshing dynamics of fluid systems in microgravity was precisely given by Hung and Pan $[12,13]$. Interested rearlers are suggested to refer to these references $[12,13]$. The computational algorithms applicable to cryogenic fluid under microgravity are also given in our earlier studies [3-5]. In this study, to show a realistic case, an example of a full-scale GP-B spacecraft dewar tank with an outer radius of $68 \mathrm{~cm}$, an inner radius of $13 \mathrm{~cm}$, top and bottom dome radius of $80 \mathrm{~cm}$ and a height of $145 \mathrm{~cm}$ has 

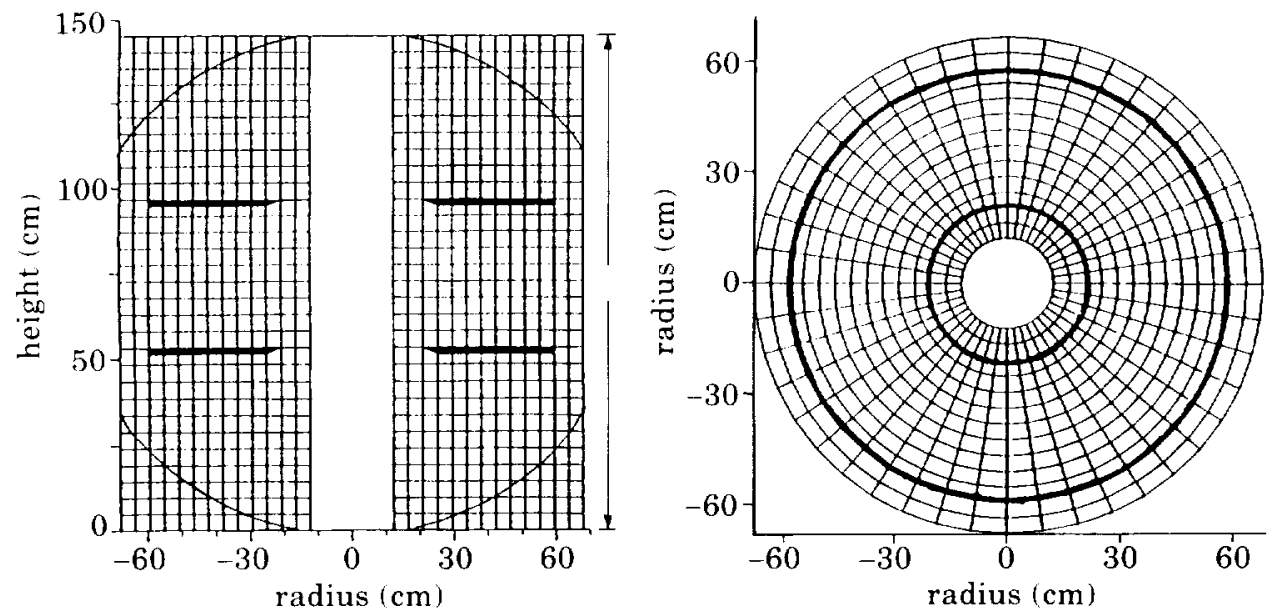

Fig. 2. - Distribution of grid points in the $a$ ) radial-axial plane at $\theta=0^{\circ}$, and $b$ ) the radialcircumferential plane at $z=L / 2$ of the cylindrical coordinate for propellant dewar tank.

been used in the numerical simulation. The dewar tank is $80 \%$ filled with cryogenic liquid helium and the rest of the ullage is filled with helium vapor. The temperature of cryogenic helium is $1.8 \mathrm{~K}$. In this study the following data were used: liquid-helium density $=0.146 \mathrm{~g} / \mathrm{cm}^{3}$, helium vapor density $=0.00147 \mathrm{~g} / \mathrm{cm}^{3}$, fluid pressure $=1.66 \times 10^{4}$ dyne $/ \mathrm{cm}^{2}$, surface tension coefficient at the interface between liquid helium and helium vapor $=0.353 \mathrm{dyne} / \mathrm{cm}$, liquid-helium viscosity coefficient $=$ $=9.61 \times 10^{-5} \mathrm{~cm}^{2} / \mathrm{s}$; and contact angle $=0^{\circ}$. The initial profiles of the liquid-vapor interface for the rotating dewar are determined from computations based on algorithms developed for the steady-state formulation of microgravity fluid management [3-5].

A staggered grid for the velocity components is used in this computer program. The MAC (marker-and-cell) method for studying fluid flows along a free surface is adopted [14]. The finite-difference method employed in this numerical study was the "Hybrid Scheme" [15]. This method is valid for any arbitrary interface location between the grid points and is not limited to middle-point interfaces [16]. An algorithm for a semi-implicit method[17] was used for modeling the flow field. The time step is determined automatically based on the size of the grid points and the velocity of flow fields. More than 200 iterations have been applied for each time step to assure the convergence of series. As to the volume conservation of liquid, a deviation of less than $1 \%$ error is always guaranteed before moving to the next time step. A detailed description of the computational algorithm applicable to microgravity fluid management is illustrated in our earlier studies [3-5]. Figures $2 a$ ) and $b$ ) show the distribution of grid points for the dewar tank in the radial-axial plane and radial-circumferential plane, respectively, in cylindrical coordinates.

Baffle boards illustrated in fig. 1 are not a final design of GP-B dewar container. The figure is rather a description of artist view of the cross-section of dewar. In this study, two pieces of baffle boards are given as an example to investigate the possible effect of baffle on the damping of slosh wave excitation. Results of this study will be used as the guidelines for the future design of the baffle to the equipped in the GP-B dewar. 


\section{3. - Dynamical behavior of liquid-vapor interface disturbances driven by gravity gradient acceleration.}

For the GP-B Spacecraft, as an example, which is an Earth satellite orbiting at $650 \mathrm{~km}$ altitude directly over the poles, the orbit period, $\tau_{0}$ can be computed from the following expression $[1,9]$ :

$$
\tau_{0}=2 \pi \frac{R_{\mathrm{c}}^{3 / 2}}{R_{\mathrm{E}} g_{0}^{1 / 2}},
$$

where $R_{\mathrm{E}}$ denotes the radius of the Earth ( $\left.=6373 \mathrm{~km}\right) ; R_{\mathrm{c}}$ the radius of the circular orbit $\left(=R_{\mathrm{E}}+h=7023 \mathrm{~km}\right) ; h$ the orbit altitude $(=650 \mathrm{~km}) ;$ and $g_{0}$ the Earth gravity acceleration $\left(=9.81 \mathrm{~m} / \mathrm{s}^{2}\right)$. The orbital period is $\tau_{0}=97.6 \mathrm{~min}$, and the orbital rate is $n=2 \pi / \tau_{0}=1.07 \cdot 10^{-3} \mathrm{rad} / \mathrm{s}$.

The gravity gradient acceleration $\hat{a}_{\mathrm{gg}}$ acting on the fluid mass of the spacecraft can be shown to be[10,11,13]

$$
\hat{a}_{\mathrm{gg}}=-n^{2}\left[3\left(\hat{r}_{\mathrm{c}} \cdot \hat{d}\right) \hat{r}_{\mathrm{c}}-\bar{d}\right],
$$

where $\hat{d}$ is the vector (not a unit vector) from the fluid element to the spacecraft mass center; $\hat{r}_{\mathrm{c}}$ a unit vector from the spacecraft mass center to the center of the Earth; and $n$ the orbit rate.

For the GP-B Spacecraft, as an example, it is assumed that the gravity gradient exerted on the mass center of the spacecraft orbiting around the Earth is zero[1]. In order words, all the gravity acceleration exerted on the fluid elements in the dewar is nothing but the gravity gradient acceleration which is defined in eq. (8) [1,9-11]. In this study, we are interested in investigating how the gravity gradient acceleration affects the dynamical behaviors of the cryogenic fluid elements of helium.

For convenience of mathematical calculation, let us describe all the parameters involved in eq. (8) in terms of Cartesian coordinates. In order to match with the computer simulation, mathematical derivation are considered in the first quadrant. Figure 3 illustrates the geometrical relationship of the parameters shown in eq. (8).

The gravity gradient acceleration acting on the fluid element located at $(r, \theta, z)$ can be transformed into the cylindrical, non-inertial frame of coordinates as follows [13]:

$$
\begin{aligned}
\hat{a}_{\mathrm{gg}}= & \left(\begin{array}{c}
a_{\mathrm{gg}, r} \\
a_{\mathrm{gg}, \theta} \\
a_{\mathrm{gg}, z}
\end{array}\right)=-n^{2}\left(\begin{array}{ccc}
\cos (\theta+\omega t) & \sin (\theta+\omega t) & 0 \\
-\sin (\theta+\omega t) & \cos (\theta+\omega t) & 0 \\
0 & 0 & 1
\end{array}\right) \\
& \cdot\left(\begin{array}{c}
3\left[(z-L / 2) \cos \psi_{\mathrm{E}}-r \cos (\theta+\omega t) \cdot \sin \psi_{\mathrm{E}}\right] \sin \psi_{\mathrm{E}}+r \cos (\theta+\omega t) \\
r \sin (\theta+\omega t) \\
-3\left[(z-L / 2) \cos \psi_{\mathrm{E}}-r \cos (\theta+\omega t) \cdot \sin \psi_{\mathrm{E}}\right] \cos \psi_{\mathrm{E}}+(z-L / 2)
\end{array}\right),
\end{aligned}
$$

where $\psi_{\mathrm{E}}$ denotes the azimuth angle of the Earth toward the location of the spacecraft mass center, or angle between the spacecraft rotating axis and radial direction from the spacecraft mass center to the center of the Earth; $n$ the orbit rate; and $t$ the time. 


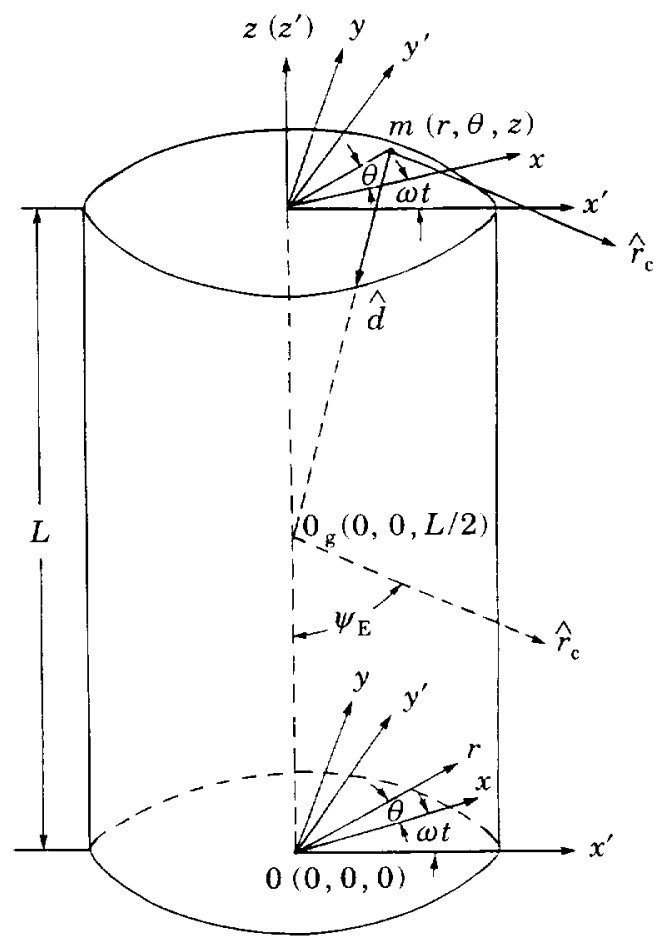

Fig. 3. - Coordinate system for the computation of gravity gradient acceleration, $0_{\mathrm{g}}$ is the location of geometric center of cylinder. The direction of $\bar{r}_{c}$ points toward Earth center. $\left(x^{\prime}, y^{\prime}, z^{\prime}\right)$ denotes the absolute coordinate system, while $(x, y, z)$ illustrates the non-inertial spacecraft-bound, coordinate system.

At time $t=0$, the rotating axis of the spacecraft is aligned with the radial direction from the Earth center to the spacecraft mass center. It is assumed that the spacecraft axis of rotation is linearly turning around from $0^{\circ}$ to $360^{\circ}$ in $600 \mathrm{~s}$ when the spacecraft is orbiting around the Earth. Thus, $\psi_{E}$ can be defined as

$$
\psi_{\mathrm{E}}=2 \frac{\pi}{\tau} t
$$

where $\tau$ is the spacecraft gravity turn-around time $(=600 \mathrm{~s}$ in this case); and $t$ is the time measured from the instant when the direction of the spacecraft rotating axis is aligned with the radial direction of the spacecraft mass center to the center of the Earth.

31. Liquid-vapor interface oscillations in a rotating dewar without baffle-boards. - The equilibrium shape of the liquid-vapor interface for a rotating dewar without baffle boards under a background gravity environment of $10^{-7} g_{0}$ and rotating speed of 0.1 r.p.m. is a doughnut configuration with a near circular kidney-shaped cross-section according to the computations based on the numerical algorithm developed in our earlier studies [3-5]. Figure 4a) shows the initial shape of interface in the $(r, z)$-plane at $\theta=0^{\circ}$ and $180^{\circ}$; fig. $\left.4 b\right)$ shows the initial profile of liquid-vapor 

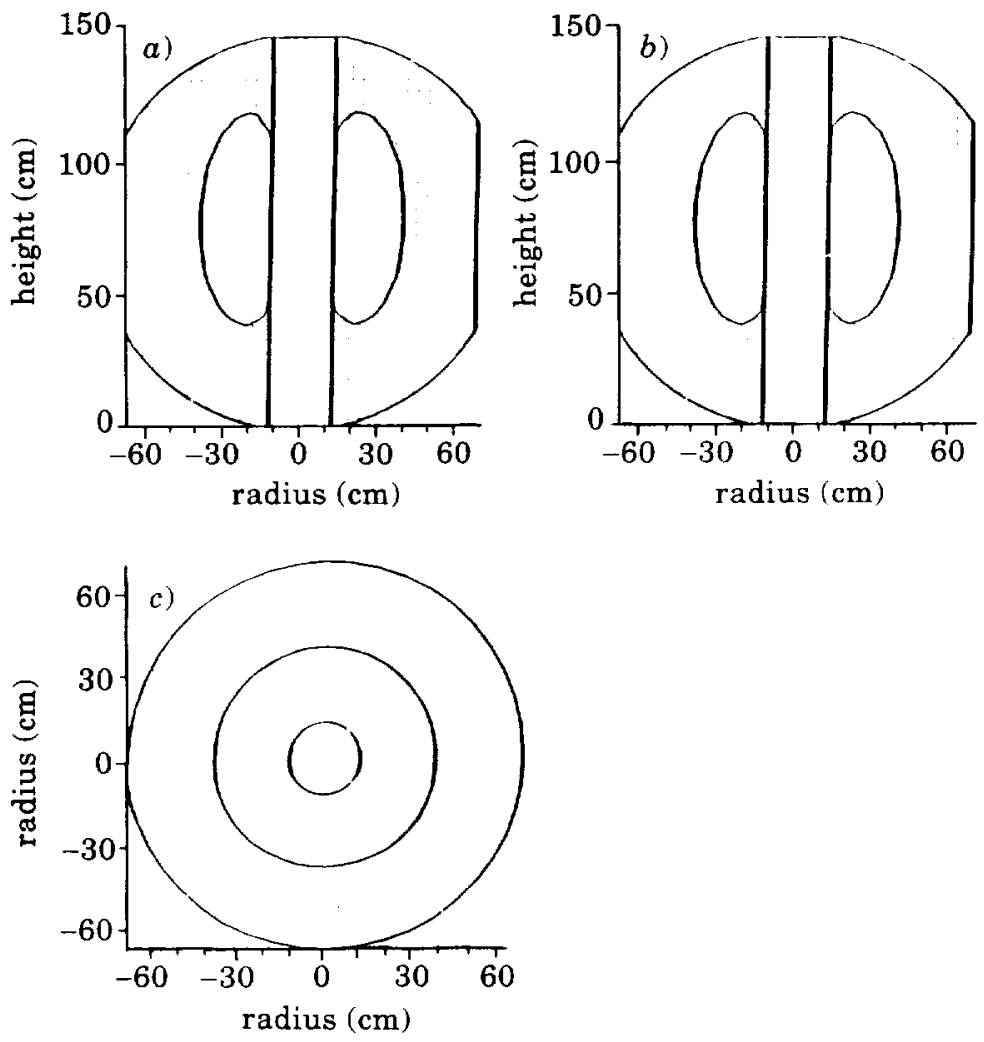

Fig. 4. - Initial profiles of liquid-vapor interface for Gravity Probe-B module with the rotating dewar without baffles under background gravity of $10^{7} g_{0}$, rotating speed of 0.1 r.p.m. and direction of background gravity $\left.\psi_{\mathrm{t}}=0^{\circ} . a\right)$ In the $(r, z)$-plane at $\theta=0^{\circ}$ and $\left.180^{\circ}, b\right)$ in the $(r, z)$-plane at $\theta=90^{\circ}$ and $270^{\circ}$, and $\left.c\right)$ in the $(r, \theta)$-plane at $z=91.8 \mathrm{~cm}$.

interface in the $(r, z)$-plane at $\theta=90^{\circ}$ and $270^{\circ}$; and fig. $\left.4 c\right)$ shows the initial profile of the liquid-vapor interface in the $(r, \theta)$-plane at height $z=91.8 \mathrm{~cm}$.

Figures 5 and 6 show the time evolution of the dynamical behavior of the liquid-vapor interface oscillations driven by the gravity gradient acceleration acting on a rotating dewar without baffle boards. For the convenience of comparison, the figures of liquid-vapor interface profiles were chosen with the same values of the time. The six figures of the time sequence chosen throughout this paper are at times $t=60,150,240,450,510$ and $600 \mathrm{~s}$. They clearly show a series of asymmetric oscillations excited along the surface of liquid-vapor interface driven by asymmetric gravity gradient acceleration acting on rotating dewar without baffle boards.

Careful examination of gravity gradient equations, shown in eqs. (8) and (9), indicate that there are greater positive components of acceleration longitudinal to the direction aligned with spacecraft mass center to the Earth center, and smaller negative components of acceleration transverse to this direction. As we indicated in eq. (10), the angle $\psi_{\mathrm{E}}$ varies with time. This phenomon shows that gravity gradient acceleration exerted on the spacecraft is equivalent to the combination of a time-dependent twisting force with a turn-around direction and torsional moment 


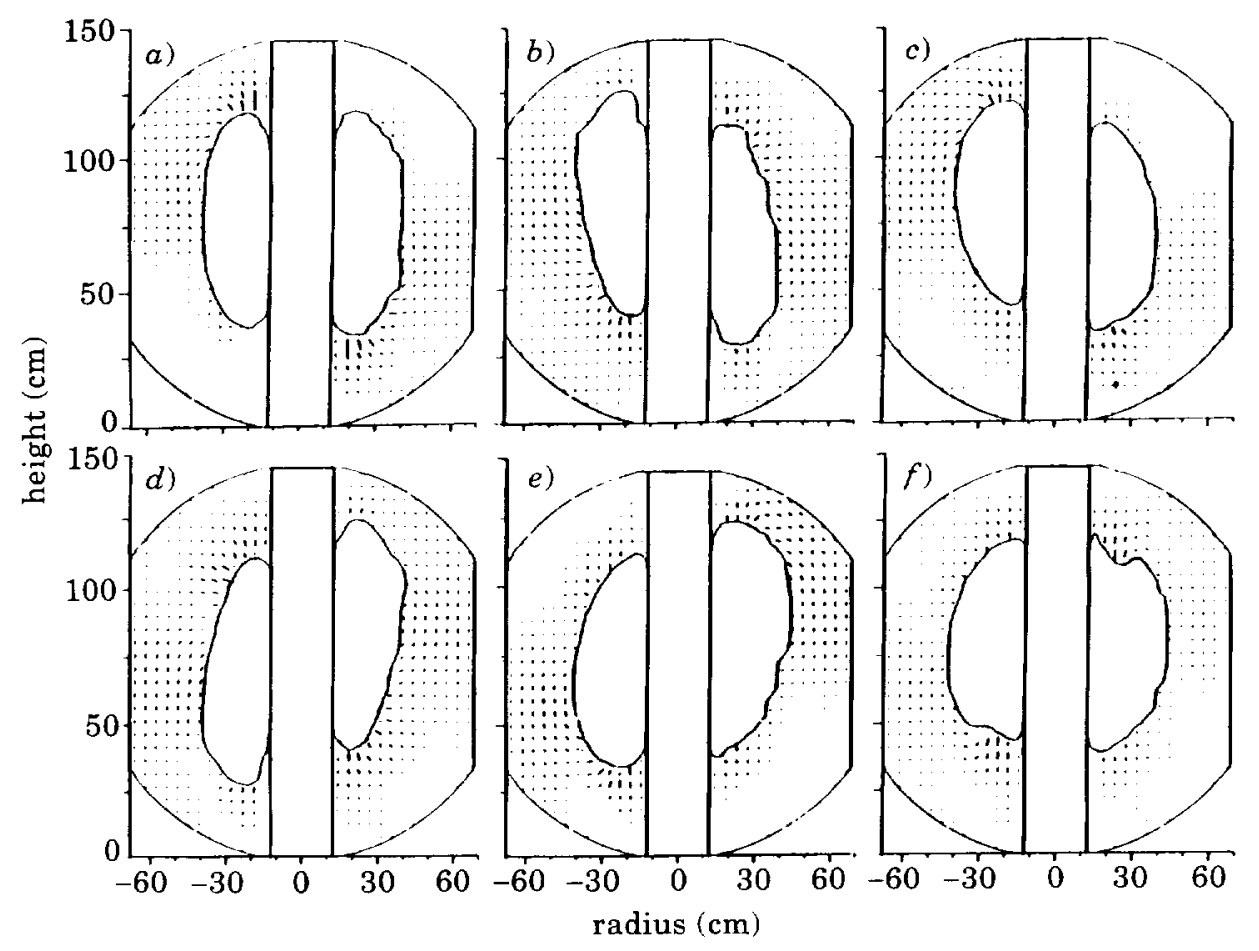

Fig. 5. - Time evolution of liquid-vapor interface oscillations, driven by gravity gradient acceleration for a rotating dewar without baffle, in the $(r, z)$-plane at $\theta=0^{\circ}$ and $180^{\circ}$. The rotating speed of dewar is 0.1 r.p.m., and the period of the gravity direction turn-around time is $600 \mathrm{~s}$. a) $t=60 \mathrm{~s}$, b) $t=150 \mathrm{~s}, c) t=240 \mathrm{~s}, d$ ) $t=450 \mathrm{~s}, e) t=510 \mathrm{~s}, f) t=600 \mathrm{~s}$.

acting on the spacecraft, when it is orbiting around the Earth. It is shown in fig. 5 and 6 that the deformation of the bubble is created by an asymmetric torsional moment with a twisting force.

Figure 5 shows the time sequence of the liquid-vapor interface profiles, driven by the gravity gradient acceleration, in the vertical $(r, z)$-plane at $\theta=0^{\circ}$ and $180^{\circ}$. It indicates that the doughnut-shaped bubbles (helium vapor) change from an axially symmetric to an asymmetric one on a plane aligned with the vector of the gravity gradient acceleration, as $\psi_{\mathrm{E}}$ varies from $0^{\circ}$ to $360^{\circ}$.

One-up one-down and one-down one-up oscillations of two bubbles with a doughnut cross-section in the vertical $(r, z)$-plane, shown in fig. 5 , indicate a very important characteristic of gravity gradient acceleration which produces a combination of time-dependent equivalent of torsional moment and twisting force acting on the spacecraft when it is orbiting around the Earth.

Figure 6 shows the time evolution of the liquid-vapor interface oscillations driven by the same gravity gradient acceleration, as that shown in figure 5 , in the horizontal $(r, \theta)$-plane for heights of $91.8 \mathrm{~cm}$. It shows that a hollow-cylinder configuration of the liquid-vapor interface changes from axial symmetry (see fig. 4c)) to asymmetry (see fig. 6). These figures also show the eccentric contour of the bubble cross-section which oscillates and rotates along clockwise direction with respect to time. These results coincide with our previous description for the time evolution of liquid-vapor 


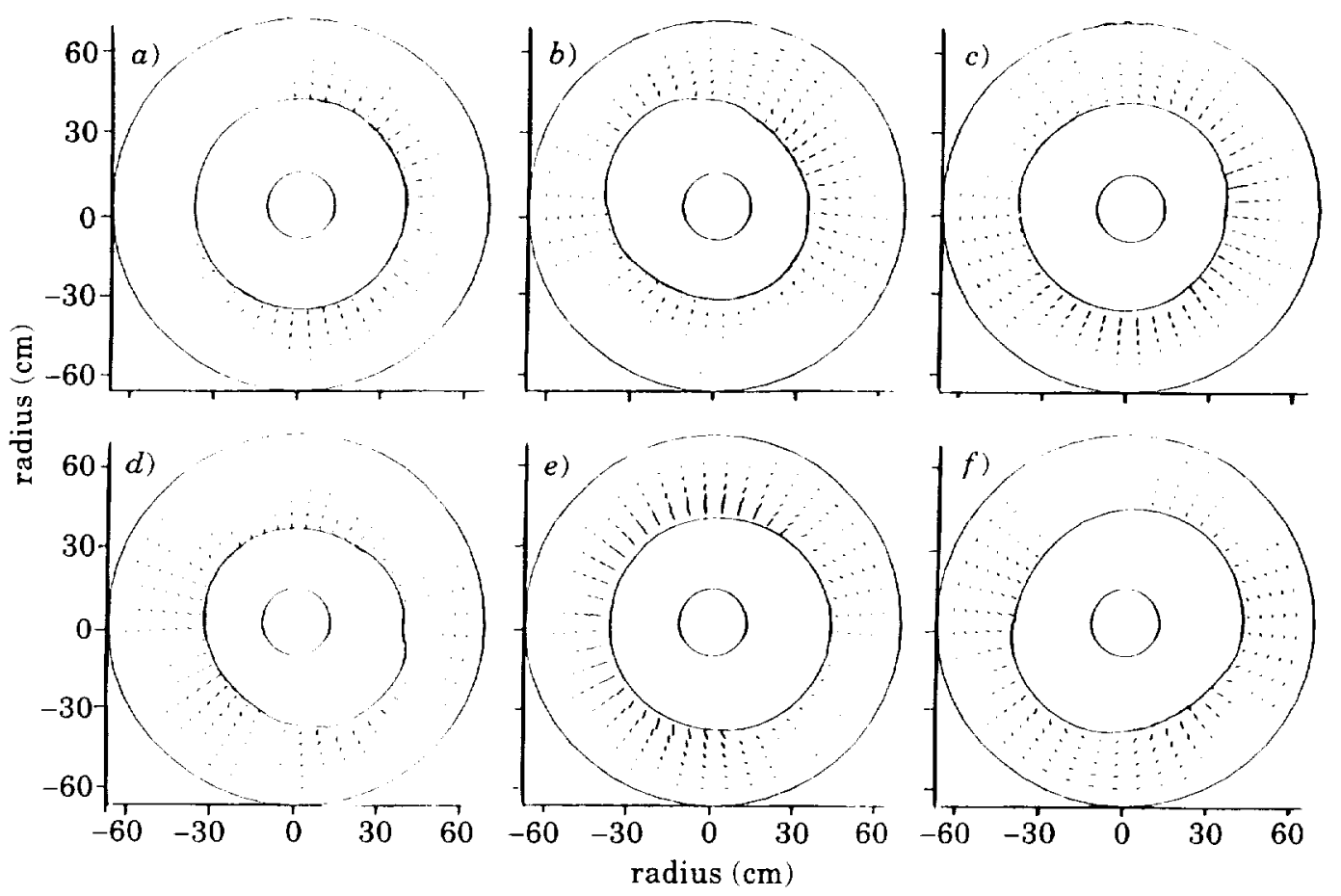

Fig. 6. - Time evolution of liquid-vapor interface oscillations driven by gravity gradient acceleration, for a rotating dewar without baffles, in the $(r, \theta)$-plane at height $z=91.8 \mathrm{~cm}$. The rotating speed of dewar is 0.1 r.p.m. and the period of gravity direction turn-around is $600 \mathrm{~s}$. From $a$ ) to $f$ ) same $t$ values as fig. 5 .

interface in the vertical $(r, z)$-plane, which indicate that gravity gradient acceleration, as a driving force, produces an effect equivalent to a time-dependent torsional moment and twisting force acting on the spacecraft fluid system.

3'2. Liquid-vapor interface oscillations in a rotating dewar with baffles. - For the purpose of reducing the degree of asymmetry in the liquid-vapor distribution, a couple of baffle boards are installed in the dewar. They have an inner radius of $20.87 \mathrm{~cm}$, an outer radius of $59.43 \mathrm{~cm}$, and a thickness of $1.93 \mathrm{~cm}$ as an example. These two baffles are located at $z_{1}=53.16 \mathrm{~cm}$, and $z_{2}=96.67 \mathrm{~cm}$. All other conditions are similar to the case without baffle boards, discussed earlier. Figures $7 a$ ), $b$ ) and $c$ ) show the initial shape of the three-dimensional interface profile, interface in the $(r, z)$-plane at $\theta=0^{\circ}$ and $180^{\circ}$, interface profile in the $(r, z)$-plane at $\theta=90^{\circ}$ and $270^{\circ}$ and in the $(r, \theta)$-plane at height $z=91.8 \mathrm{~cm}$, respectively, for a rotating dewar with baffle boards.

Figures 8 and 9 show the time evolution of the dynamical behavior of the liquid-vapor interface oscillations driven by gravity gradient acceleration acting on rotating dewar with baffle boards. Comparison between fig. 5 and 8 , and between fig. 6 and 9 shows a greater degree of asymmetry for the excitation of slosh waves along the liquid-vapor interface in a rotating dewar without baffles than that with baffles. 

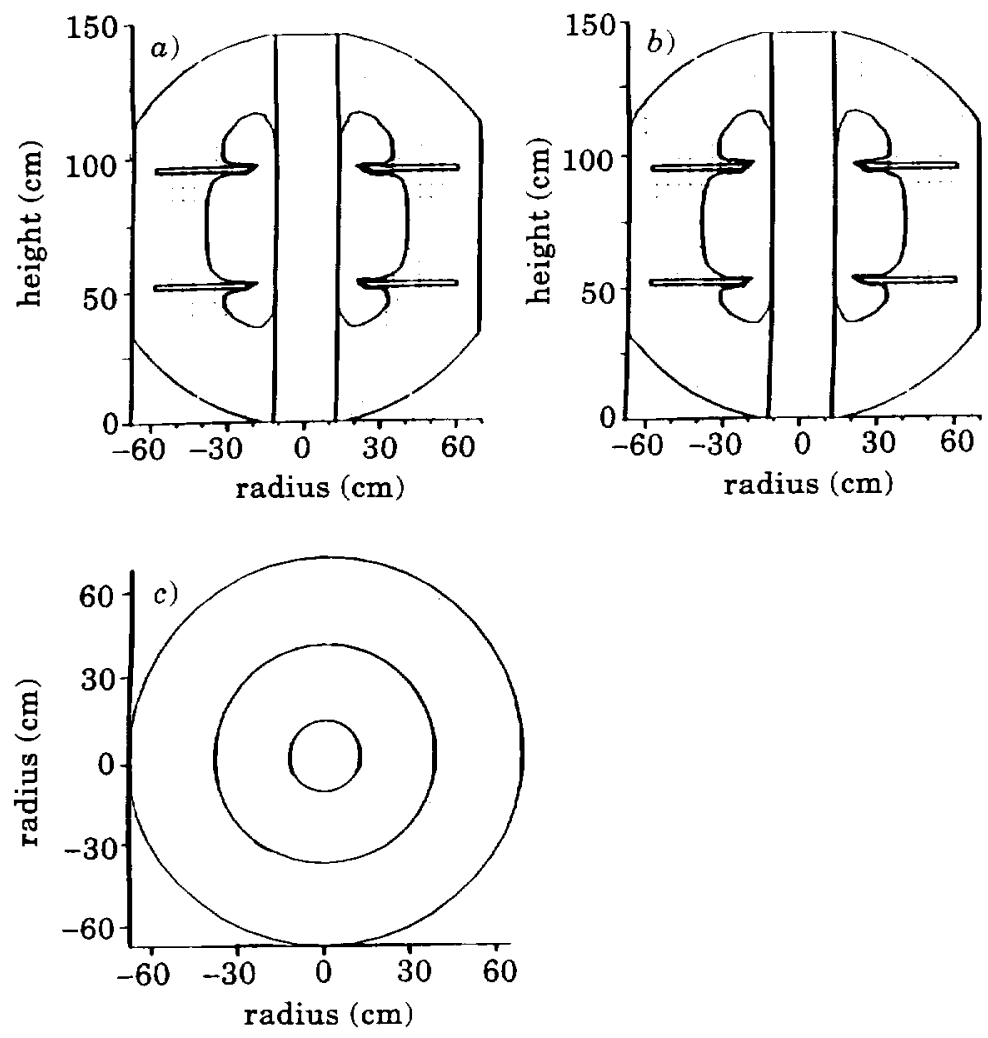

Fig. 7. - Initial profile of liquid-vapor interface for Gravity Probe-B module with the rotating dewar with baffle boards under background gravity of $10^{-7} g_{0}$, rotating speed of 0.1 r.p.m. and direction of background gravity $\psi_{\mathrm{E}}=0^{\circ}$. a) In the $(r, z)$-plane at $\theta=0^{\circ}$ and $\left.180^{\circ}, b\right)$ in the $(r, z)$-plane at $\theta=90^{\circ}$ and $270^{\circ}$, and $\left.c\right)$ in $(r, \theta)$-plane at $z=91.8 \mathrm{~cm}$.

Figure 8 shows the time evolution of the dynamical behavior of the liquid-vapor interface profiles, driven by the same gravity gradient acceleration as that shown in figure 5 , in the vertical $(r, z)$-plane at $\theta=0^{\circ}$ and $180^{\circ}$, for a rotating dewar with baffle. Similar to fig. 5 , fig. 8 also shows that the doughnut-shaped bubble (helium vapor) changes configuration from axial symmetric to asymmetric at a plane aligned with the vector of the direction of background gravity and the rotating axis as $\psi_{\mathrm{E}}$ varies from $0^{\circ}$ to $360^{\circ}$ for rotating dewar with baffle. Comparison between fig. 5 and fig. 8 shows a greater degree of asymmetry for the excitation of slosh waves along the liquid-vapor interface in a rotating dewar without baffles than that with baffles.

Again one-up one-down and one-down one-up oscillations of two bubbles, partially cut by baffle boards, with the cross-section of doughnut in the vertical $(r, z)$-plane, shown in fig. 8 with baffles, indicate a very important characteristic of gravity gradient acceleration which produces an effect equivalent to a combination of time-dependent torsional moment and twisting force acting on the spacecraft when it is orbiting around the Earth.

Figure 9 shows the time evolution of the liquid-vapor interface oscillations, driven by a gravity gradient acceleration similar to that shown in figure 5 , in the horizontal 


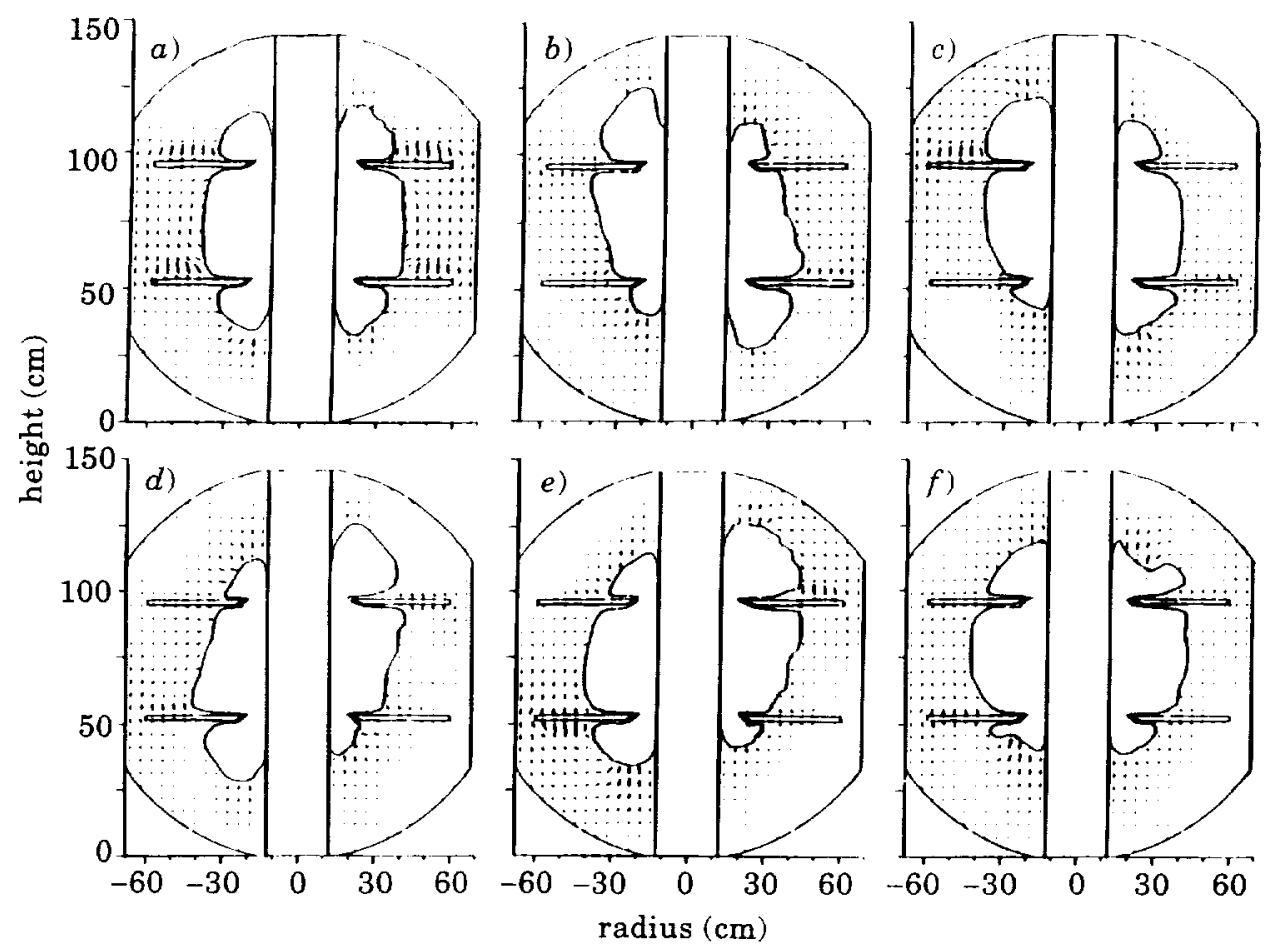

Fig. 8. - Time evolution of liquid-vapor interface oscillations driven by gravity gradient acceleration for a rotating dewar with baffles in the $(r, z)$-plane at $\theta=0^{\circ}$ and $180^{\circ}$. The period of gravity direction turn-around is $600 \mathrm{~s}$, and the rotating speed 0.1 r.p.m. From $a$ ) to $f$ ) same $t$ values as fig. 5 .

$(r, \theta)$-plane at heights of $z=91.8 \mathrm{~cm}$. It shows that a hollow-cylinder shape configuration of the liquid-vapor interface changes from axial symmetric (figure $7 c$ )) to asymmetric profiles (fig. 9).

Time fluctuations of the locations of bubble mass centers of the rotating dewar without and with baffle boards driven by gravity gradient acceleration have been computed. The evolution of $x_{\mathrm{c}}, y_{\mathrm{c}}$ and $z_{\mathrm{c}}$ denote the time-dependent variations of the locations of bubble mass centers along the $x, y$ and $z$ axes, respectively.

The values of bubble mass center fluctuations for rotating dewar without baffle driven by gravity gradient acceleration are $\left(\Delta x_{\mathrm{c}}, \Delta y_{\mathrm{c}}, \Delta z_{\mathrm{c}}\right)=(3.2,3.7,3.0) \mathrm{cm}$, while that of the bubble mass center fluctuations with baffle are $\left(\Delta x_{\mathrm{c}}, \Delta y_{\mathrm{c}}, \Delta z_{\mathrm{c}}\right)=$ $=(2.2,2.8,2.1) \mathrm{cm}$. Comparison between bubble mass center fluctuations driven by gravity gradient acceleration without and with baffle provides the following conclusions: a) Torsional-moment- and twisting-force-equivalent gravity gradient acceleration exerted on the rotating dewar container produces greater values of fluctuations for rotating dewar without baffle than that with baffle. $b$ ) Dynamies of bubble (liquid-vapor interface) driven by torsional-moment- and twistingforce-equivalent gravity gradient produce bubble mass center fluctuations with values of $\left.\Delta y_{\mathrm{c}}>\Delta x_{\mathrm{c}}>\Delta z_{\mathrm{c}} . c\right)$ Comparison of the values of bubble mass center fluctuations without and with baffle shows that the fluctuations of bubble mass center induced by gravity gradient acceleration for dewar without baffle are greater than 


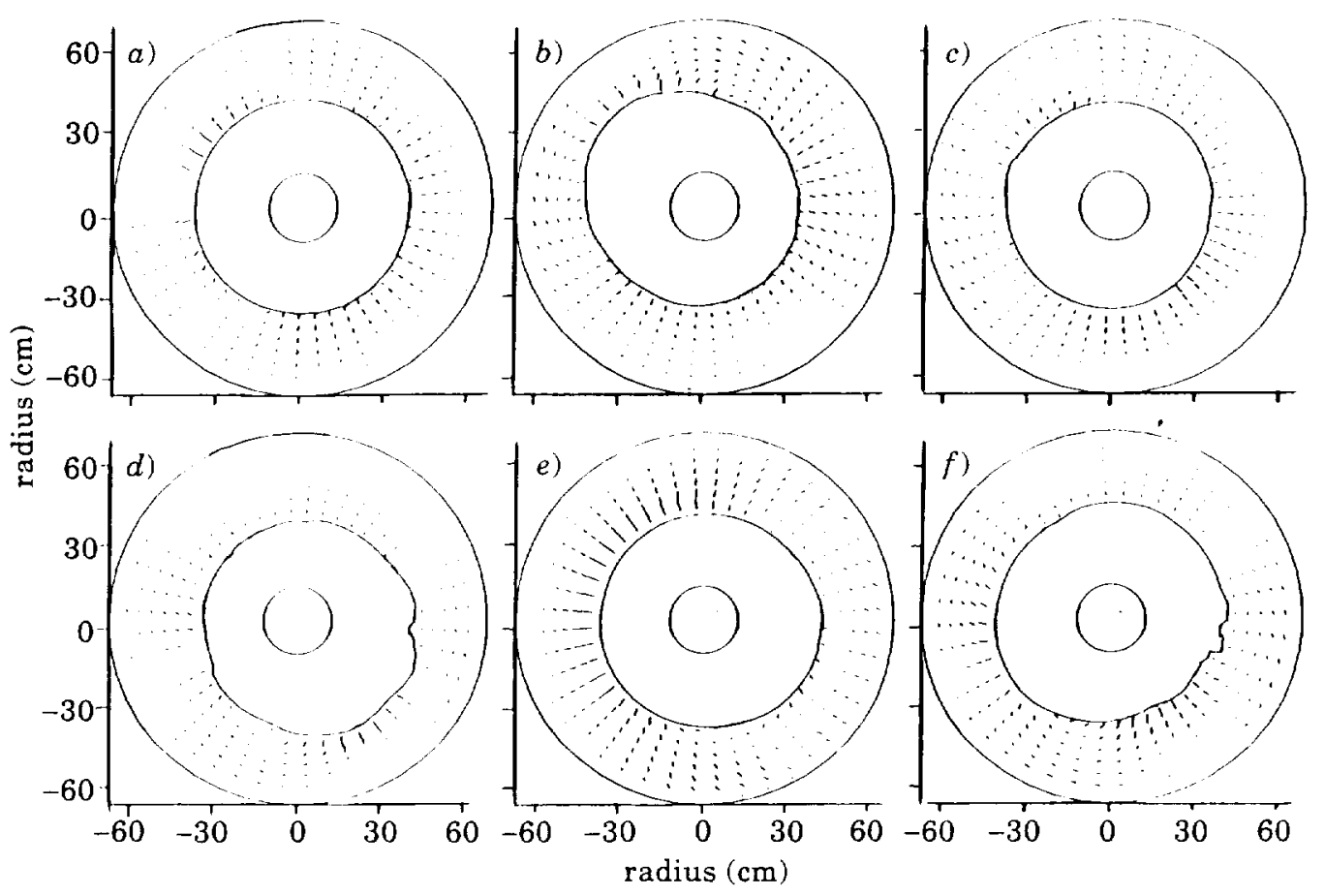

Fig. 9. - Time evolution of liquid-vapor interface oscillations driven by gravity gradient acceleration for a rotating dewar with baffles in the $(r, \theta)$-plane at height $z=91.8 \mathrm{~cm}$. The period of gravity direction turn-around is $600 \mathrm{~s}$, and the rotating speed of 0.1 r.p.m. From $a$ ) to $f$ ) same $t$ values as fig. 5 .

those induced by dewar with baffle. $d$ ) Fluctuations of both $x_{\mathrm{c}}$ and $y_{\mathrm{c}}$ start from zero while that of $z_{\mathrm{c}}$ starts from a non-zero value at the middle point of the height of partially filled liquid container.

\section{4. - Characteristics of slosh-wave-induced fluctuations in fluid-system moment and angular momentum.}

Slosh-wave-induced fluctuations in the fluid system of rotating dewar introduce time-dependent disturbances in moment and angular momentum of the spacecraft propulsion system. In addition to the angular velocity along the rolling axis, there are induced angular velocities along the yawing and pitching axes due to the fluid motion inside the rotating container. These angular velocities in yawing and pitching axes, caused by the fluid flows in a partially liquid-filled container, readjust the angular velocity of the rolling axis.

Mathematical formulations of time-dependent disturbances in moment and angular momentum of the spacecraft propulsion system due to slosh-wave-induced fluctuations in the fluid system of the rotating dewar are given by Hung et al. $[18,19]$. By using these mathematical derivations [18-20], one can compute slosh-waveinduced fluctuations in fluid system and angular momentum.

Figure 10a) shows the computed time variation of the fluctuations of angular 


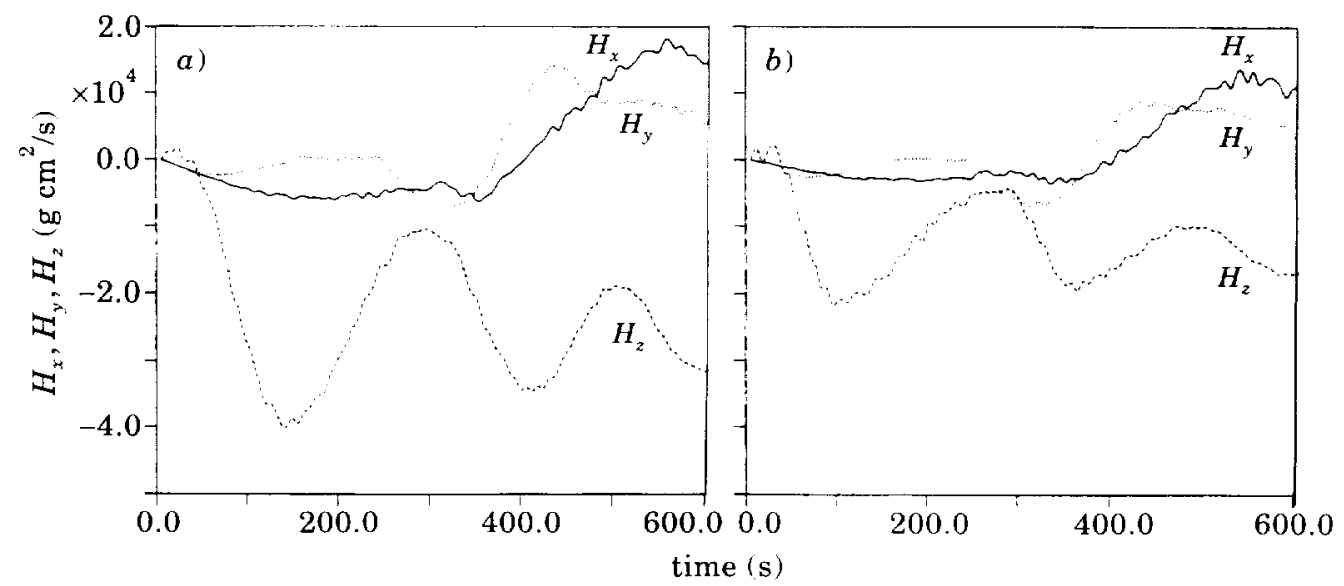

Fig. 10. - Time sequence of angular momentum fluctuations $\left(H_{x}, H_{y}, H_{z}\right)$ due to the slosh wave excitation driven by gravity gradient acceleration in a rotating dewar with speed of 0.1 r.p.m. and gravity turn-around time of $600 \mathrm{~s}$. a) For angular-momentum fluctuations in a rotating dewar without baffle board. b) For angular-momentum fluctuations in a rotating dewar with baffle boards.

momentum driven by gravity gradient acceleration for rotating dewar without baffle boards. This figure shows the following results: $a$ ) The values of angularmomentum fluctuations are $\left(\Delta H_{x}, \Delta H_{y}, \Delta H_{z}\right)=(25475,21203,41530) \mathrm{g} \mathrm{cm}^{2} / \mathrm{s}$; it clearly indicates $\Delta H_{z}>\Delta H_{x}>\Delta H_{y}$. The maximum absolute values of angular momentum are $\operatorname{Max}\left(\left|H_{x}\right|,\left|H_{y}\right|,\left|H_{z}\right|\right)=(17886,13953,39974) \mathrm{g} \mathrm{cm}^{2} / \mathrm{s}$. b) The initial values of $H_{x}, H_{y}$ and $H_{z}$ start from zero in the non-inertial frame while that of the $H_{z}$ starts from non-zero value in the inertial frame. This is due to the fact that $H_{z}$ starts with a rotating speed of 0.1 r.p.m. while there is no angular velocity along the $x$ and $y$ axes at the initial point in the inertial frame. c) Variations of oscillation frequency of $H_{z}$ are very much similar to the diurnal variation of planetary atmospheres, while the variations of oscillation frequency of $H_{x}$ and $H_{y}$ are very much similar to the meridional and zonal variations of planetary atmospheres.

Figures $(1 a), b$ ) and $c$ ) show variations of fluid moments due to slosh waves driven by gravity gradient acceleration for a rotating dewar without baffle boards along the $x, y$ and $z$ axes, respectively. The values of fluid moment fluctuations are $\left(\Delta M_{x}, \Delta M_{y}, \Delta M_{z}\right)=(680,558,928)$ dyne $\mathrm{cm}$. The maximum absolute values of fluid moment are $\operatorname{Max}\left(\left|M_{x}\right|,\left|M_{y}\right|,\left|M_{z}\right|\right)=(441,300,547)$ dyne $\mathrm{cm}$. It is shown that $\Delta M_{z}>\Delta M_{x}>\Delta M_{y}$ and $\left|M_{z}\right|>\left|M_{x}\right|>\left|M_{y}\right|$. The characteristics of the fluctuations of fluid moments driven by gravity gradient acceleration for a rotating dewar without baffle boards draw three-point conclusions similar to those drawn for the fluctuations of angular momentum of a fluid system for a rotating dewar without baffle boards shown earlier.

Figure $10 b$ ) shows the computed time variation of the fluctuations of angular momentum driven by gravity gradient acceleration for a rotating dewar with baffle boards. The values of angular momentum fluctuations are $\left(\Delta H_{x}, \Delta H_{y}, \Delta H_{z}\right)=$ $=(17599,15917,27615) \mathrm{g} \mathrm{cm}^{2} / \mathrm{s}$ and the maximum absolute values of angular momentum are $\operatorname{Max}\left(\left|H_{x}\right|,\left|H_{y}\right|,\left|H_{z}\right|\right)=(12957,9433,26715) \mathrm{g} \mathrm{cm}^{2} / \mathrm{s}$. It is shown 

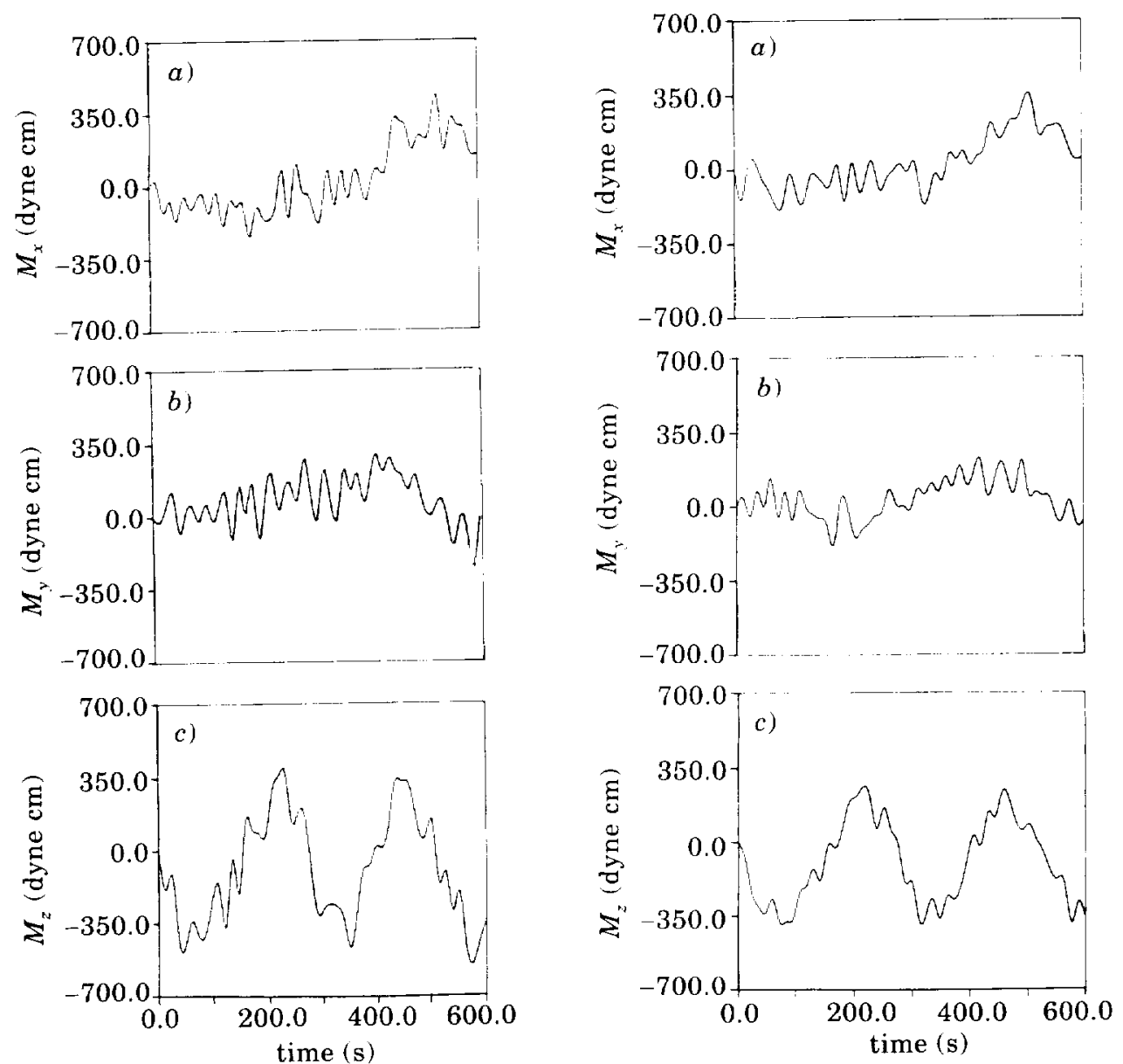

Fig. 11.

Fig. 12.

Fig. 11. - Time sequences of fluid moment fluctuations caused by slosh wave excitation driven by gravity gradient acceleration for rotating dewar without baffle boards in rotation speed of 0.1 r.p.m. and gravity turn-around time of $600 \mathrm{~s}$. $a$ ) For moment along $x$-direction; $b$ ) for moment along $y$-direction; and $c$ ) for moment along $z$-direction.

Fig. 12. - Time sequences of fluid moment fluctuations caused by slosh wave excitation driven by gravity gradient acceleration for rotating dewar with baffle boards, in a rotation speed of 0.1 r.p.m. and gravity turn-around time of $600 \mathrm{~s}$. $a$ ) For moment along $x$-direction; $b$ ) for moment along $y$-direction; and $c$ ) for moment along $z$-direction.

that $\Delta H_{z}>\Delta H_{x}>\Delta H_{y}$ and $\left|H_{z}\right|>\left|H_{x}\right|>\left|H_{y}\right|$. Comparison between fig. 10a) and b) for angular-momentum fluctuations driven by gravity gradient acceleration for a rotating dewar with and without baffle boards shows that they are of the same order, and it also shows that gravity-gradient-acceleration-excited values and fluctuations of angular momentum for a rotating dewar without baffle boards are of greater magnitudes than those driven by gravity gradient acceleration, for a rotating dewar with baffle boards. 
Figures $12 a), b$ ) and c) show variations of fluid moments due to slosh waves excited by gravity gradient acceleration for a rotating dewar with baffle boards along the $x, y$ and $z$ axes, respectively. The values of fluid moment fluctuations are $\left(\Delta M_{x}, \Delta M_{y}, \Delta M_{z}\right)=(495,408,645)$ dyne $\mathrm{cm}$ and the maximum absolute values of fluid moment are $\operatorname{Max}\left(\left|M_{x}\right|,\left|M_{y}\right|,\left|M_{z}\right|\right)=(329,232,382)$ dyne $\mathrm{cm}$. It is shown that $\Delta M_{z}>\Delta M_{x}>\Delta M_{y}$ and $\left|M_{z}\right|>\left|M_{x}\right|>\left|M_{y}\right|$. The characteristics of the fluctuations of fluid moments driven by gravity gradient acceleration for rotating dewar with baffle boards draw three-point conclusions similar to those drawn for the fluctuations of angular momentum of fluid systems driven by gravity gradient acceleration for a rotating dewar without baffle boards shown earlier. Comparison between fig. 11 and 12 for fluid momentum fluctuations driven by gravity gradient acceleration for a rotating dewar with and without baffle boards shows that they are of the same order, and it also shows that gravity-gradient-acceleration-excited values and fluctuations of fluid moment for a rotating dewar without baffle boards are of greater magnitudes than those driven by gravity gradient acceleration, for a rotating dewar with baffle boards.

\section{5. - Discussion and conclusion.}

In this study, the spacecraft gravity turn-around time is assumed to be $600 \mathrm{~s}$ which is the same as the time period of the spacecraft axis of rotation. The effect equivalent to a combination of twisting force and torsional moment of gravity gradient acceleration, acting on the rotating dewar with and without baffle boards when it is orbiting around the Earth, induces one side-up and the other side-down motions in the bubble cross-section with doughnut profiles, in the vertical $(r, z)$-plane, and an eccentric contour of bubble in the horizontal cross-section in the $(r, \theta)$-plane which oscillates and rotates with a clockwise direction with respect to time. It is shown that slosh-wave-excited liquid-vapor interface fluctuations, driven by gravity gradient acceleration for a rotating dewar without baffle boards produce greater asymmetry disturbances than those driven by gravity gradient acceleration for a rotating dewar with baffle boards.

Induced angular velocities along the yawing and pitching axes caused by slosh wave excitation in partially liquid-filled container, in addition to the existing rotating motion along the rolling axis, have been investigated. Results show that there are a series of large-amplitude fluctuations of angular momenta and fluid moments along yawing and pitching axes. In particular, slosh waves excited by the asymmetry gravity gradient acceleration contribute greatly to the large-amplitude fluctuations of angular momentum and fluid moment along the rolling axis of the spacecraft.

As a viscous force, between liquid and solid interface, greatly contributes to the damping effect of slosh wave excitation, a rotating dewar with baffle provides more areas of liquid-solid interface than that of the rotating dewar without baffles. It is shown that a rotating dewar equipped with baffle boards greatly reduce the fluctuations of both angular momentum and fluid system moment than that without baffle board.

Time-dependent variations in slosh-wave-excited liquid-vapor interface fluctuations driven by gravity gradient acceleration will change and modify the asymmetry distribution of liquid-vapor in the rotating dewar container. This means that both moment and angular momentum of spacecraft will be deeply disturbed by the 


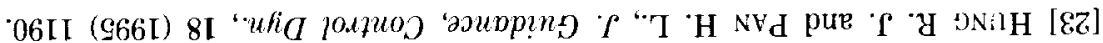

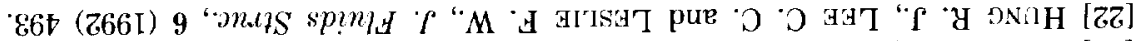

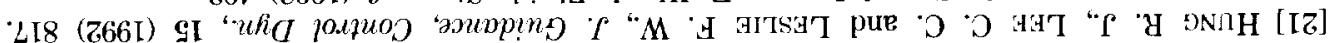
28I

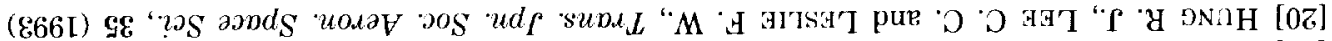
LIZ (

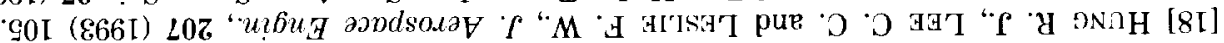

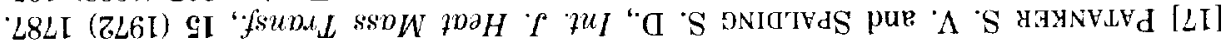

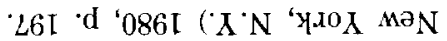

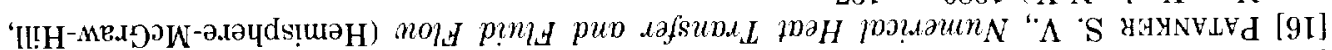

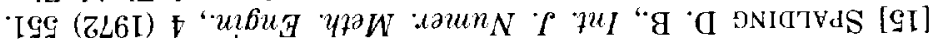

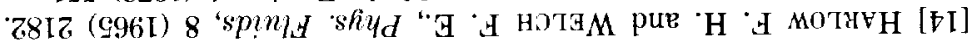

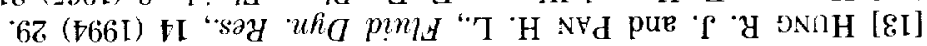

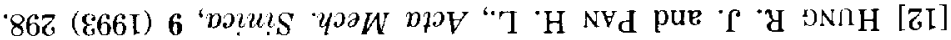
'6LZI-I dd '8L6I (oos!oub.

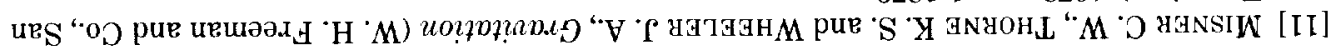

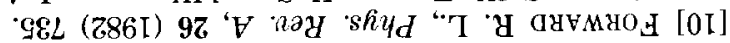
†86L (MOJSOK

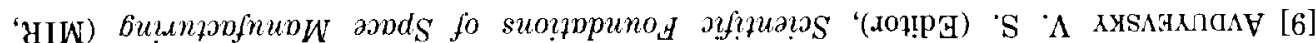

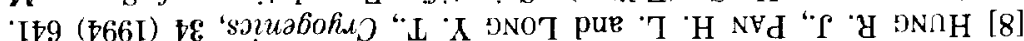

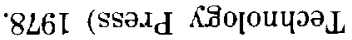

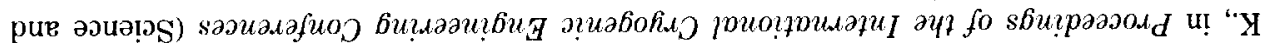

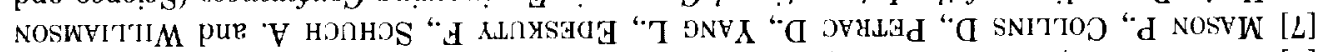

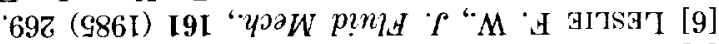

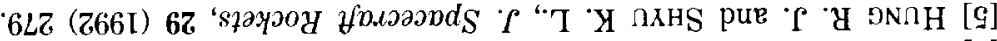

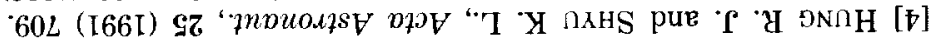

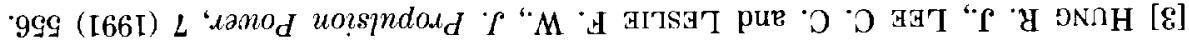

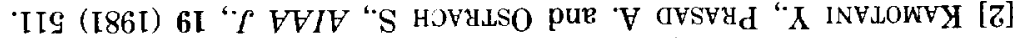

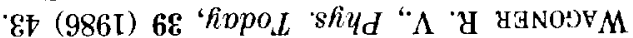

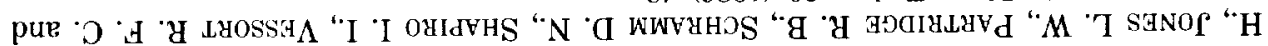

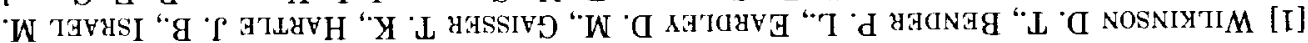

SGDNGYAGGY

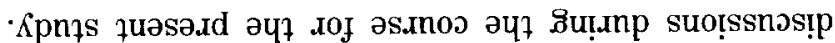

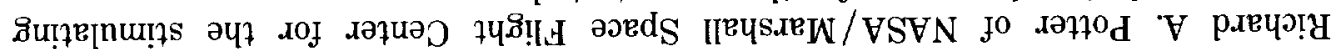

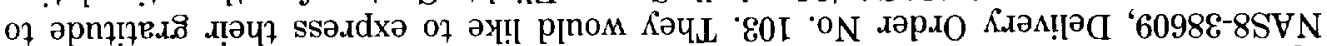

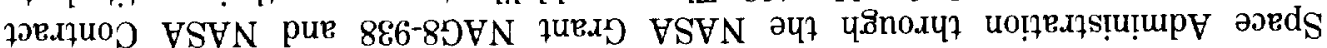

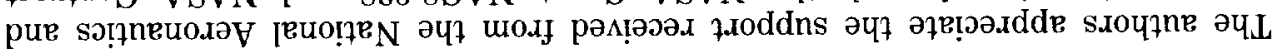

$\cdot[8 z-[z]$ suo!̣

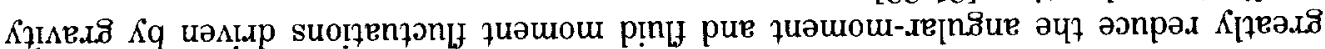

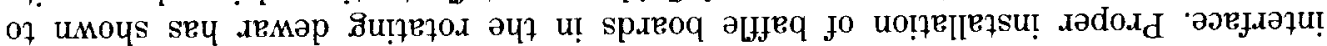

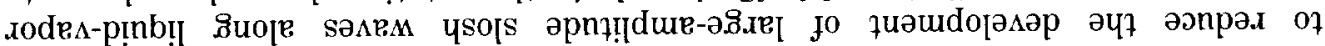

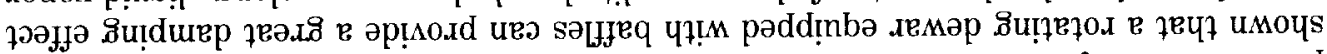

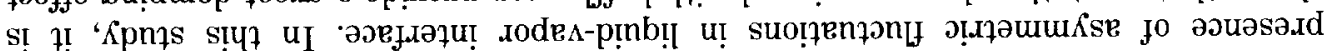


